\title{
Patient controlled analgesia was more effective than nurse controlled analgesia after cardiac surgery
}

Gust R, Pecher S, Gust A, et al. Effect of patient-controlled analgesia on pulmonary complications after coronary artery bypass grafting. Crit Care Med 1999 Oct;27:2218-23.

QUESTION: In patients who have had elective coronary artery bypass grafting, is patient controlled analgesia (PCA), alone or combined with non-steroidal anti-inflammatory drugs, as effective as nurse controlled analgesia for pulmonary complications and quality of pain control?

\section{Design}

Randomised (allocation concealed), blinded (outcome assessor), controlled trial with 3 days of follow up.

\section{Setting}

A university medical centre in Germany.

\section{Patients}

120 patients (mean age 61 y, $84 \%$ men) who had had elective coronary artery bypass grafting. Exclusion criteria were severe underlying disease, need for catecholaminergic support after extubation of the trachea, neurological deficit preventing assessment, return to the operating room, or use of antidepressants and class 1 antiarrhythmics. Follow up was complete.

\section{Intervention}

Patients received education about pain assessment and use of PCA before surgery. All care except pain management was the same. After extubation, patients were allocated to PCA, PCA and indomethacin, or nurse controlled analgesia (40 in each group) for up to 3 days. Intravenous piritramide, $1.5 \mathrm{mg}$ per bolus with a 10 minute lockout interval and no background infusion, was delivered using the PCA device. Indomethacin, $50 \mathrm{mg}$ was given rectally 3 times per day. The nurse administered analgesia included titrated piritramide up to $7.5 \mathrm{mg}$, indomethacin in $50 \mathrm{mg}$ suppositories, or both.

\section{Main outcome measures}

New areas of atelectasis, infiltrates, or air bronchograms as detected by daily anteroposterior chest radiographs. Pain sensation measured using a visual analogue scale, satisfaction with pain control, and side effects (nausea and vomiting, excessive sedation, urinary retention, and confusion) were secondary outcome measures.

\footnotetext{
Main results

Patients in the 2 PCA groups used more piritramide than did those in the nurse controlled group $(\mathrm{p}<0.05)$ : for the PCA alone group, daily use was 24, 20, and $8 \mathrm{mg}$ for days 1, 2, and 3; for the PCA plus indomethacin group, daily use was 22,15 , and $10 \mathrm{mg}$; and for the nurse controlled group, daily use was 11,5 , and $2 \mathrm{mg}$. More patients in PCA groups had lower atelectasis scores (fewer lung complications) and felt their pain management was excellent $(28 \%$ and $35 \% v 3 \%)$ by day 3 $(\mathrm{p}<0.05)$ than in the nurse controlled group. By day 3 the visual analogue pain scale scores were lower in the 2 PCA groups (32 for PCA alone, 35 for PCA and indomethacin, and 43 for nurse controlled analgesia, $\mathrm{p}<0.05$ for both PCA groups compared with nurse
}

controlled analgesia). The groups did not differ for side effects. No patients developed pneumonia or returned to the intensive care unit with pulmonary or pain related complications.

\section{Conclusion}

Patient controlled analgesia, with or without indomethacin, was more effective than nurse controlled analgesia for reduction of early pulmonary complications, pain control, and satisfaction with pain management.

\section{COMMENTARY}

The benefits of PCA for relieving pain and increasing patient satisfaction are established. ${ }^{1}$ However, investigations into other effects tend to concentrate on adverse events, such as nausea and vomiting. Studies of the effect of good pain control on pulmonary complications have been limited, and yet this is advocated as a potential benefit of this method of analgesia.

One reason for this limitation might be the method used to detect signs of pulmonary complications. Gust $e t$ al used a simple but effective method of detecting pulmonary complications, assessment of chest radiographs for early signs of atelectasis. They were able to show that PCA with or without non-steroidal anti-inflammatory drugs reduced the incidence of this complication in their population of patients who had cardiac surgery.

The use of radiologists who were blinded to treatment group to assess chest radiographs for signs of atelectasis was a strength of this study. None of the patients fulfilled $\geqslant 3$ of the criteria for the diagnosis of pneumonia. The authors explain that the low incidence of pneumonia in their study might be because of the short follow up period of 3 days or the more strict criteria they used to diagnose pneumonia. Despite this, they assert that, as atelectasis is a precursor of more serious events, PCA can prevent serious pulmonary complications. The study used piritamide, an opioid of limited availability outside Europe. It has similar properties to morphine but a slightly longer duration of action.

This study is of relevance to nurses working in the fields of acute pain management and critical care, especially cardiothoracic surgery, as well as nurses working in other surgical areas. It increases our knowledge of the potential benefits of PCA, specifically the reduction of pulmonary complications after surgery. This is clinically important because it provides evidence on the potential for PCA to reduce postoperative complications other than pain.

Gareth Parsons, RGN, MSc Clinical Nurse Specialist, Acute Pain Anaesthetic Department, Princess of Wales Hospital Bridgend, South Wales, UK

1 Ballantyne JC, Carr DB, Chalmers TC, et al. Postoperative patient-controlled analgesia: meta-analyses of initial randomized control trials. J Clin Anesth 1993;5:182-93. 